\title{
CORRELATION OF VELOCITY DISTRIBUTIONS IN AN ANCHOR-AGITATED VESSEL USING A BICUBIC B-SPLINE FUNCTION
}

\author{
KUnIO ARAI, KoJI TAKAHASHI AND SHOZABURo SAITO \\ Department of Chemical Engineering, Tohoku University, Sendai 980
}

\begin{abstract}
A surface-fitting method has been developed for the velocity data scattered on a given surface by using a bicubic B-spline function and is applied to data obtained from the analysis of the path lines of tracer particles in an anchor-agitated vessel.

This method has been proved to be of great use for expression of the velocity distributions throughout an agitated vessel if sufficient data for path lines are available.
\end{abstract}

\section{Introduction}

Detailed information on velocity distributions in an agitated vessel is very helpful to an understanding of mixing performance, since convective transport tends to be the rate-controlling step in the mixing of highly viscous liquids. Although velocity distributions for highly viscous liquids have been investigated experimentally by many authors in various mixer geometries ${ }^{3,5,11-13)}$, they are too complicated to be expressed quantitatively over the entire flow field in the agitated vessel. However, this difficulty may be partially eliminated if a sufficient number of discrete velocity data is available for any given surface. In this case, the data can be treated as two-dimensional and may be correlated by an appropriate surfacefitting method. Therefore, two problems should be solved to describe three-dimensional velocity distributions quantitatively. One is that of obtaining the velocity data for any specified surface and the other that of developing a surface-fitting method for data whose underlying function may be very complicated. One of the best ways of obtaining such data is to measure the path lines throughout the agitated vessel as conducted by Kamiwano et al..$^{9}$ and the author $\mathrm{s}^{101}$, because the velocity data for any specified surface can be given at the intersections of the path lines and the surface.

In this work, a surface-fitting method for the velocity data scattered on a given surface has been developed with the use of a bicubic B-spline function and applied to the velocity data ${ }^{10\rangle}$ obtained from the measurements of the path lines of tracer particles in an anchoragitated vessel.

Received February 6, 1952. Correspondence concerning this article should be addressed to $\mathrm{S}$. Saito.

\section{A Surface-Fitting Method of Velocity Distributions}

1. 1 Approximating function for least squares surface fittings

In this work, a cubic B-spline function ${ }^{7,14,16\rangle}$ is employed as an approximating function, because of its success in applications to the correlations of complicated two-dimensional data ${ }^{16)}$. The approximating problem is confined to an anchor-agitated vessel because the numerical solutions and experimental data of the velocity distributions have been presented by the author $\mathrm{s}^{10)}$. The method developed in this work may be easily extended to other geometries. The flow field for an anchor-agitated vessel is shown in Fig. 1. Using the polar co-ordinate system, the bicubic B-spline function is expressed as:

$$
S_{i}(r, \theta, c)=\sum_{j=1}^{p+4} \sum_{k=1}^{q+4} c_{j, k} M_{j}(r) N_{k}(\theta)
$$

where the parameter vector of coefficients, $\boldsymbol{c}$, is represented by:

$$
\boldsymbol{c}=\left(c_{1,1}, c_{2,1}, \cdots, c_{p+4,1}, \cdots, c_{j, k}, \cdots, c_{p+4, q+4}\right)^{T}
$$

and the solution is to determine the parameter vector, $c$, by minimizing the sum of squares of residuals, $Q$, and simultaneously satisfying the given boundary

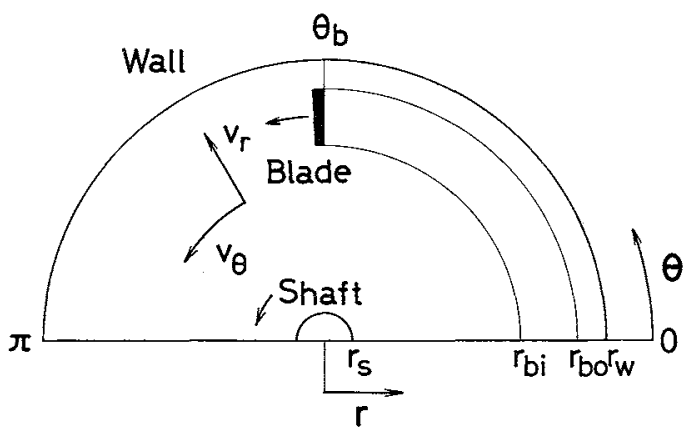

Fig. 1 Flow field in anchor-agitated vessel 


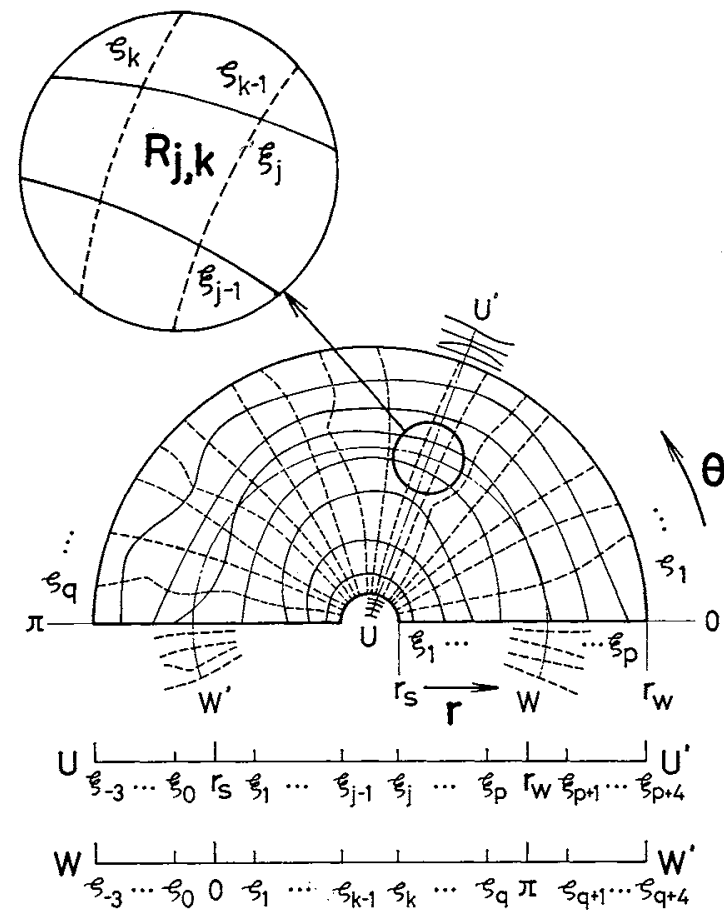

Fig. 2 Schematic diagram of random grid for surface fitting with use of cubic B-spline function

values as follows:

$$
\operatorname{minimize}\left[Q=\sum_{d=1}^{m}\left\{S_{i}\left(r_{d}, \theta_{d}, \boldsymbol{c}\right)-V_{i, d}\right\}^{2}\right]
$$

and

$$
\left.S_{i}(r, \theta, c)\right|_{\mid \text {on boundaries }}=\text { (given values) }
$$

where $V_{i, d}$ denotes the experimental value of the velocity in $i$-direction at point $\left(r_{d}, \theta_{d}\right) . M_{j}$ and $N_{k}$ in Eq. (1) are one-dimensional normalized cubic Bsplines in $r$ and $\theta$-directions represented by the recurrence relation respectively ${ }^{2,6)}$ :

$$
L_{j}(x)=\left(\lambda_{j}-\lambda_{j-4}\right) B_{4, j}(x) \quad a \leq x \leq b
$$

where

$$
\begin{aligned}
& B_{n, j}(x)=\frac{\left(x-\lambda_{j-n}\right) B_{n-1, j-1}(x)+\left(\lambda_{j}-x\right) B_{n-1, j}(x)}{\lambda_{j}-\lambda_{j-n}} \\
& B_{1, j}(x)=\left\{\begin{array}{cl}
\left(\lambda_{j}-\lambda_{j-1}\right)^{-1} & \left(\lambda_{j-1} \leqq x<\lambda_{j}\right) \\
0 & \text { (otherwise) }
\end{array}\right.
\end{aligned}
$$

$\lambda_{j}$ is called a knot located on $x$-direction where $a<\lambda_{1}<$ $\cdots<\lambda_{j-1}<\lambda_{j}<\cdots<\lambda_{h}<b$. The equations show that a cubic B-spline $L_{j}(x)$ is a piecewise cubic polynominal which is defined uniquely with the five knots of $\lambda_{j-4}$ to $\lambda_{j}$ and is non-zero only over four adjacent intervals between the knots of $\lambda_{j-4}$ to $\lambda_{j}$. To define the full set of B-splines in the region $a \leq x \leq b$, it is necessary to introduce eight additional knots which satisfy the following equations.

$$
\begin{aligned}
& \lambda_{-3}<\lambda_{-2}<\lambda_{-1}<\lambda_{0} \leq a \\
& b \leq \lambda_{h+1}<\lambda_{h+2}<\lambda_{h+3}<\lambda_{h+4}
\end{aligned}
$$

The first step in the least squares surface fitting problem, therefore, is to fix a grid on the plane which consists of two sets of lines, $\xi_{j}$ and $\zeta_{k}$, as shown in Fig. 2. The lines, $\xi_{-3}, \cdots, \xi_{j}, \cdots, \xi_{p+4}$, represent the locations of the knots in $r$-direction and the lines, $\zeta_{-3}, \cdots, \zeta_{k}, \cdots, \zeta_{q+4}$, those in $\theta$-direction, where

$$
\begin{aligned}
& \xi_{-3}<\cdots<\xi_{0} \leq r_{s}<\xi_{1}<\xi_{2}<\cdots<\xi_{j}<\cdots \\
& \quad<\xi_{p}<r_{w} \leq \xi_{p+1}<\cdots<\xi_{p+4}
\end{aligned}
$$

and

$$
\begin{gathered}
\zeta_{-3}<\cdots<\zeta_{0} \leq 0<\zeta_{1}<\zeta_{2}<\cdots<\zeta_{k}<\cdots \\
<\zeta_{q}<\pi \leq \zeta_{q+1}<\cdots<\zeta_{q+4}
\end{gathered}
$$

The sets of lines form a random grid partitioning the flow field into the regions, $R_{1,1}, R_{1,2}, \cdots, R_{j, k}$, $\cdots, R_{p+1, q+1}$, while region $R_{j, k}$ denotes the region which is bounded by the four lines $\xi_{j}, \xi_{j-1}, \zeta_{k}$ and $\zeta_{k-1}$.

Removing zero elements from Eq. (1), it can be simplified as follows:

$$
\begin{aligned}
& S_{i}(r, \theta, c)=\sum_{f=j}^{j+3} \sum_{g=k}^{k+3} c_{f, g} M_{f}(r) N_{g}(\theta) \\
& \quad(r, \theta) \in R_{j, k}(j=1,2, \cdots, p+1 \quad k=1,2, \cdots, q+1)
\end{aligned}
$$

where $M_{f}(r)$ is given uniquely if the five intersections of each line $\xi_{f}, \xi_{f-1}, \xi_{f-2}, \xi_{f-3}$ and $\xi_{f-4}$ with the straight line from the origin with the slope of $\tan \theta$ are known, and $N_{g}(\theta)$ if those of each line $\zeta_{g}, \zeta_{g-1}, \zeta_{g-2}, \zeta_{g-3}$ and $\zeta_{g-4}$ with the circle of radius $r$ with its center at the origin are known.

\subsection{Boundary conditions and grid generation}

When a rotating polar co-ordinate system fixed on the impeller is used, the approximating function must satisfy the following boundary conditions:

$$
\begin{aligned}
& S_{r}\left(r_{s}, \theta, \boldsymbol{c}\right)=S_{\theta}\left(r_{s}, \theta, \boldsymbol{c}\right)=0 \\
& S_{r}\left(r_{b i} \leqq r \leqq r_{b o}, \theta_{b}, \boldsymbol{c}\right)=S_{\theta}\left(r_{b i} \leqq r \leqq r_{b o}, \theta_{b}, \boldsymbol{c}\right)=0 \\
& S_{r}\left(r_{w}, \theta, \boldsymbol{c}\right)=0 \\
& S_{\theta}\left(r_{w}, \theta, \boldsymbol{c}\right)=-r_{w} / r_{b o} \\
& S_{r}(r, \pi, \boldsymbol{c})=S_{r}(r, 0, c) \\
& S_{\theta}(r, \pi, \boldsymbol{c})=S_{\theta}(r, 0, c)
\end{aligned}
$$

Successful surface fittings will depend on grid formation. In addition to the necessary condition that a grid must be drawn so that each region $R_{j, k}$ may contain at least one datum point ${ }^{8,15)}$, it will be chosen by a combination of trial and error, experience and a general knowledge of the shape of the underlying function as indicated by the data ${ }^{7}$. The following ability of a bicubic B-spline function in expressing a discontinuity also provides information useful when a grid is being formed. Namely, making two lines $\xi_{j}$ and $\xi_{j-1}$ or $\zeta_{k}$ and $\zeta_{k-1}$ coincide will produce a discontinuity in the second derivative with respect to $r$ along the line $\xi_{j}$ or with respect to $\theta$ along the line $\zeta_{k}$. Similarly, a discontinuity in the first derivative will result from making three lines coincide, and in the function value itself from making four lines coincide. 

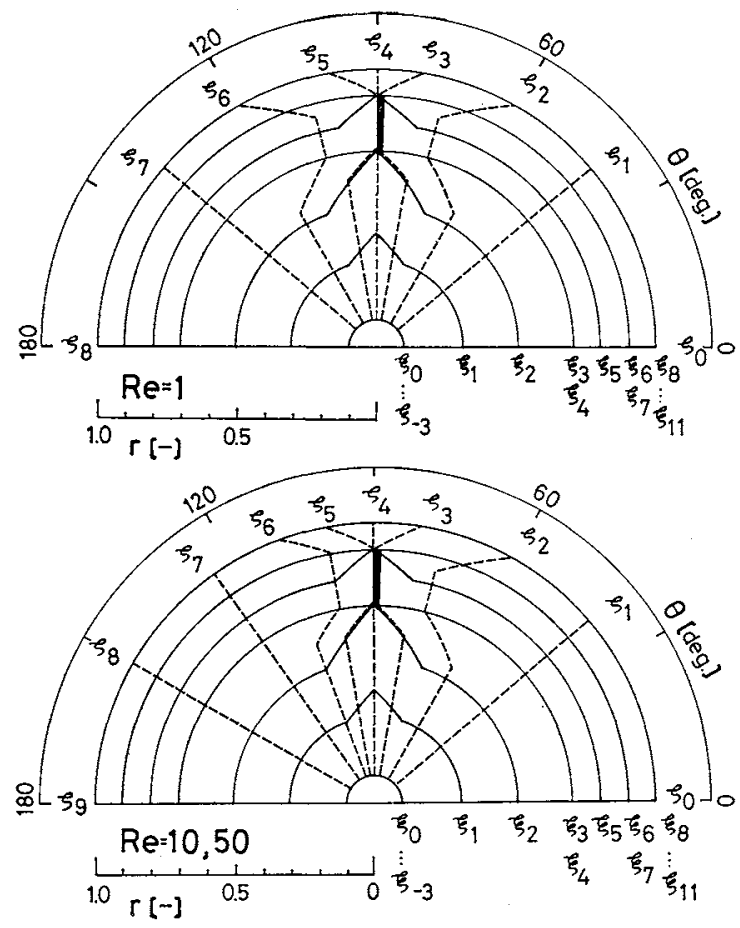

Fig. 3 Grids used for surface fittings of velocity data prepared from numerical solution

In Fig. 3, grids used for the least squares fittings of the velocity at $R e=1,10$ and 50 are shown. For numerical convenience, the additional four lines outside each end boundary of $r$-direction are made to coincide at each surface of the shaft or the wall. Namely,

$$
\begin{aligned}
& \xi_{-3}=\xi_{-2}=\xi_{-1}=\xi_{0}=r_{s} \\
& \xi_{p+1}=\xi_{p+2}=\xi_{p+3}=\xi_{p+4}=r_{w}
\end{aligned}
$$

Figure 4 (a) shows the shape of each cubic B-spline $M_{j}(r)$ near the boundaries at the shaft and the wall. As shown in the figure, only one cubic B-spline has a non-zero value on each boundary as,

$$
\begin{aligned}
& M_{j}\left(r_{s}\right)= \begin{cases}1 & \text { for } j=1 \\
0 & \text { for } j=2,3,4\end{cases} \\
& M_{j}\left(r_{w}\right)= \begin{cases}0 & \text { for } j=p+1, p+2, p+3 \\
1 & \text { for } j=p+4\end{cases}
\end{aligned}
$$

Substituting Eqs. (13) and (21) into Eq. (12) gives:

$$
\sum_{g=k}^{k+3} c_{1, g} N_{g}(\theta)=0 \text { at } r=r_{s}
$$

Since all $N_{g}(\theta)$ of $g=k$ to $k+3$ at $r=r_{s}$ have non-zero values except at the knots of $k$ to $k+3$, all the coefficients $c_{1, g}$ should be zero:

$$
c_{1, g}=0 \quad(g=1,2, \cdots, q+1)
$$

Similar results are also obtained using the boundary condition at the wall:

$c_{p+4, g}= \begin{cases}0 & \text { for radial velocity component } \\ -r_{w} / r_{b o} & \text { for tangential velocity component }\end{cases}$

$$
(g=1,2, \cdots, q+1)
$$

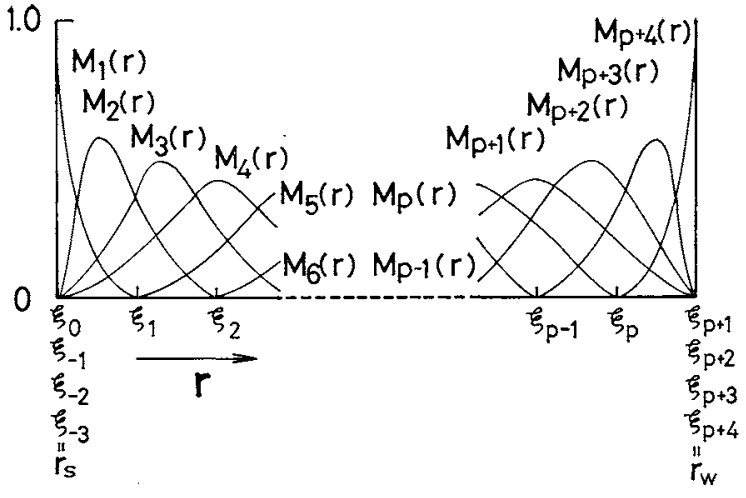

(a) at shaft and wall

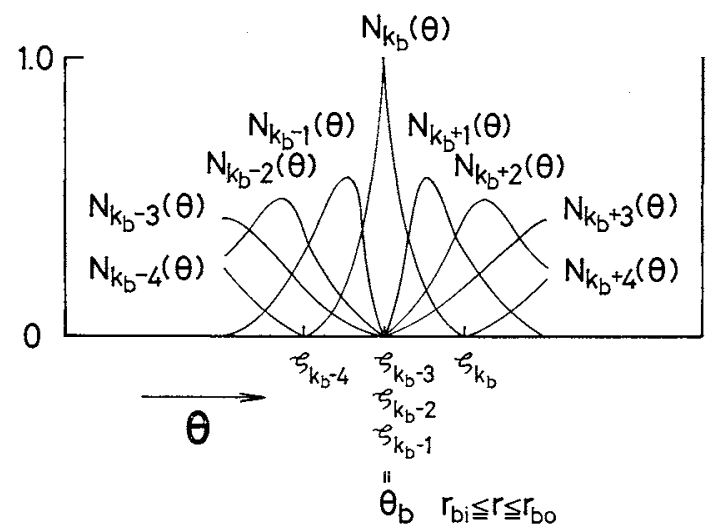

(b) at blade

Fig. 4 Graphs of each normalized cubic B-spline near solid boundaries

To satisfy the periodic condition of Eq. (17) or (18) in $\theta$-direction, the set of lines must be periodical with a period of $\pi$ radians, and this provides the following additional lines outside each end of $\theta$-direction.

$$
\begin{aligned}
& \zeta_{k}=\zeta_{q+1+k}-\pi \quad(k=0,-1,-2,-3) \\
& \zeta_{k}=\zeta_{-q-1+k}-\pi \quad(k=q+1, q+2, q+3, q+4)
\end{aligned}
$$

and the line $\xi_{j}$ must also satisfy the same periodical condition as follows:

$$
\xi_{j \text { at } \theta=0}=\xi_{j \text { at } \theta=\pi} \quad(j=0,1, \cdots, p+1)
$$

Since the first derivatives of each velocity component with respect to $r$ or $\theta$ are discontinuous at the blade, it is necessary to make three lines of $\xi$ and those of $\zeta$ coincide at the blade respectively, as shown in Fig. 3. In this figure, the two lines $\xi_{3}$ and $\xi_{4}$ or $\xi_{6}$ and $\xi_{7}$ are also made to coincide everywhere, respectively. This provides flexibility in fitting complicated data ${ }^{8)}$ although this will lead to a discontinuity in the second derivative along these lines. The shape of cubic B-spline $N_{l}(\theta)$ near the boundary of the blade is also shown in Fig. 4(b). Only one cubic B-spline has a non-zero value at the boundary as,

$$
N_{k}\left(\theta_{b}\right)= \begin{cases}1 & \text { for } k=k_{b} \\ 0 & \text { otherwise }\end{cases}
$$




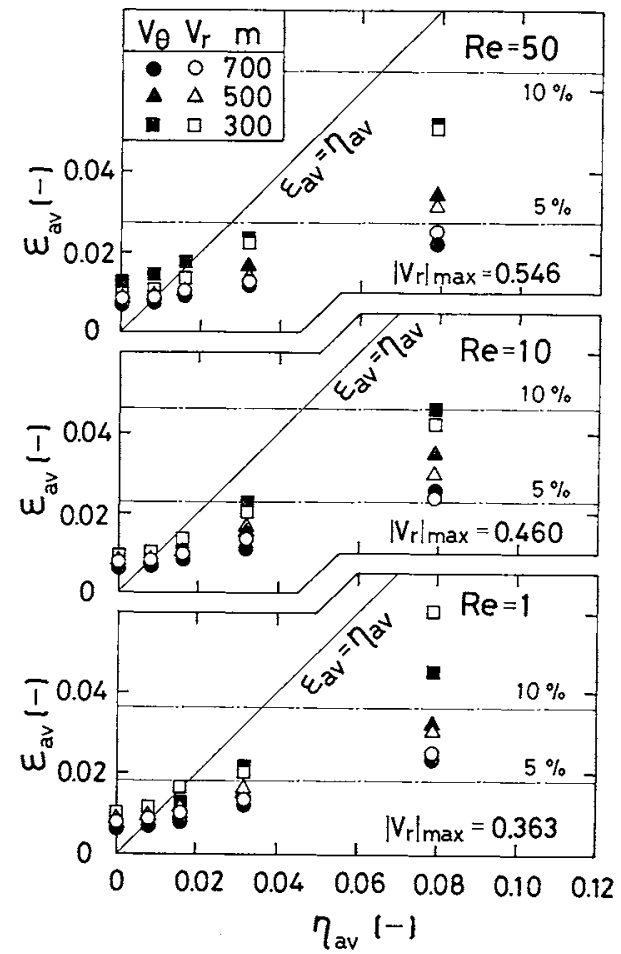

Fig. 5 Errors of surface fittings

Similarly,

$$
\begin{aligned}
& M_{j}\left(r_{b i}\right)= \begin{cases}1 & \text { for } j=j_{b i} \\
0 & \text { otherwise }\end{cases} \\
& M_{j}\left(r_{b o}\right)= \begin{cases}1 & \text { for } j=j_{b o} \\
0 & \text { otherwise }\end{cases}
\end{aligned}
$$

According to the manner in which Eq. (24) is derived, the following result is obtained from the above equations:

$$
c_{f, k_{b}}=0 \quad\left(f=\dot{j}_{b i}, \cdots, j_{b o}\right)
$$

\section{3 Normal equation}

A normal equation for solving the most probable values of the coefficients $c_{j, k}$ is given as follows:

$$
A \boldsymbol{c}=\boldsymbol{d}
$$

where $A$ is a square band matrix determined only from the values of $B$-splines at each datum point and $\boldsymbol{d}$ is a column vector determined from the values of B-splines and given data. Considering the periodic condition of Eq. (17) or (18), the cubic B-splines of $N_{q+2}(\theta)$ to $N_{q+4}(\theta)$ can be replaced by those of $N_{1}(\theta)$ to $N_{3}(\theta)$ respectively and then the rank of the matrix $A$ and the dimensions of the vector $c$ and $d$ can be reduced to $(p+4)(q+1)$. The detailed forms of $A$ and $\boldsymbol{d}$ are given in the Appendix.

Not all the components of the vector $c$ are unknown but some of them are predetermined from the boundary values as mentioned in the previous section. This constraint can be easily incorporated with the algorithm of Choleski's method for solving a normal equation ${ }^{4)}$
2. Discussion of Effectiveness of a Surface-Fitting Mehtod

\section{1 Data generation from numerical solution}

The effectiveness of a correlation method will be confirmed directly by an examination of the relationship between the errors which the velocity data contain and the deviation from the true values of the correlation values. Since it is difficult to evaluate quantitatively the errors accompanying experimental data, velocity data are prepared by the addition of pseudo experimental errors to true values as follows:

$$
V_{i, d}=v_{i}\left(r_{d}, \theta_{d}\right)+\eta_{i, d}
$$

where $v_{i}\left(r_{d}, \theta_{d}\right)$ is the true value of the velocity in $i$ direction at the point $\left(r_{d}, \theta_{d}\right)$ and is given by the numerical analysis conducted by the authors ${ }^{10)}$, and $\eta_{i, d}$ represents an error artificially generated from a random variable with a normal distribution whose mean and standard deviation are zero and $\sigma$ respectively.

\section{2 Results and discussion}

Using each grid shown in Fig. 3, least squares surface fittings were carried out for many sets of the data of radial and tangential velocities provided from Eq. (34). The true values $v_{i}\left(r_{d}, \theta_{d}\right)$ for each data set were randomly sampled from the numerical solutions for Reynolds number, $R e$, of 1,10 or $50^{10)}$. To investigate the effects of the number of data, $m$, and the magnitude of error which the data contain on the accuracy of correlation results, the data sets consisted of 300,500 or 700 data points and had an error generated from a random variable with a normal distribution whose standard deviation is $0,0.01,0.02$, 0.04 or 0.10 , respectively. In Fig. 5, the average deviation, $\varepsilon_{\mathrm{av}}$, from the true values of the correlation values is plotted against the average error, $\eta_{a v}$. $\varepsilon_{a v}$ and $\eta_{\mathrm{av}}$ are defined as:

$$
\begin{aligned}
\varepsilon_{\mathrm{av}} & =-\frac{1}{m} \sum_{d=1}^{m}\left|v_{i}\left(r_{d}, \theta_{d}\right)-S_{i}\left(r_{d}, \theta_{d}, \boldsymbol{c}\right)\right| \\
\eta_{\mathrm{av}} & =-\frac{1}{m} \sum_{d=1}^{m}\left|\eta_{i, d}\right|=-\frac{1}{m} \sum_{d=1}^{m}\left|v_{i}\left(r_{d}, \theta_{d}\right)-V_{i, d}\right| \\
& =\sqrt{\frac{2}{\pi}-\sigma}
\end{aligned}
$$

In this figure, dot-dash lines indicate 5 and $10 \%$ of the absolute maximum value of radial velocity, $\left|v_{r}\right|_{\max }$, respectively. $\left|v_{r}\right|_{\max }$ at each $R e$ is shown in the same figure and, from the definition, the maximum tangential velocity is always equal to unity.

The sources of error in correlating data are due to both fitting method and experiments. In Fig. 5, $\varepsilon_{\mathrm{av}}$ originates mainly from the former when $\eta_{\mathrm{ar}}$ approaches zero and is considered to be small enough to be practically permissible for each $R e$. On the other hand, $\varepsilon_{\mathrm{av}}$ becomes larger with increasing $\eta_{\mathrm{av}}$ 

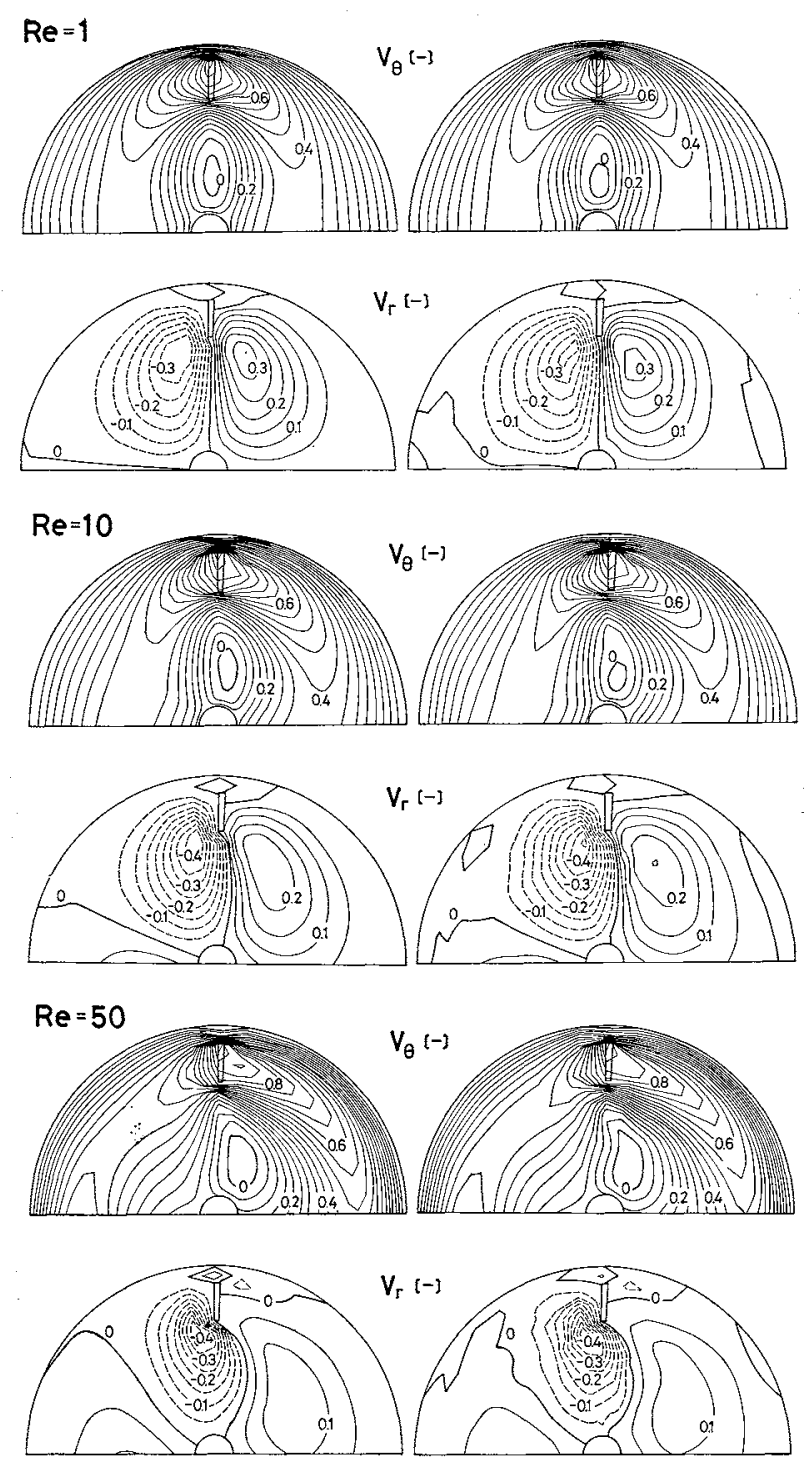

(a) numerical solution

(b) correlation results ( $m=700, \eta_{\mathrm{av}}=0.016$ )

Fig. 6 Comparisons of velocity distributions of radial and tangential velocity components for each $R e$

and this is due to the latter. It is, however, seen from the same figure that this error can be reduced when the number of data points is increased. From these facts, this method may be concluded to be successfully applicable to the correlation of velocity data. Figure 6 shows some examples of the velocity distributions for each $R e$ obtained from the correlation results and those from the numerical solution. It is evident that the correlation results and the numerical solution show quite good agreement for each Re.

\section{Application of the Surface-Fitting Method to the Observed Velocity Data in an Anchor-Agitated Vessel}

Using the experimental results reported by the authors ${ }^{10)}$, a great number of path lines in an agitated vessel are obtained as mentioned below. Co-or-

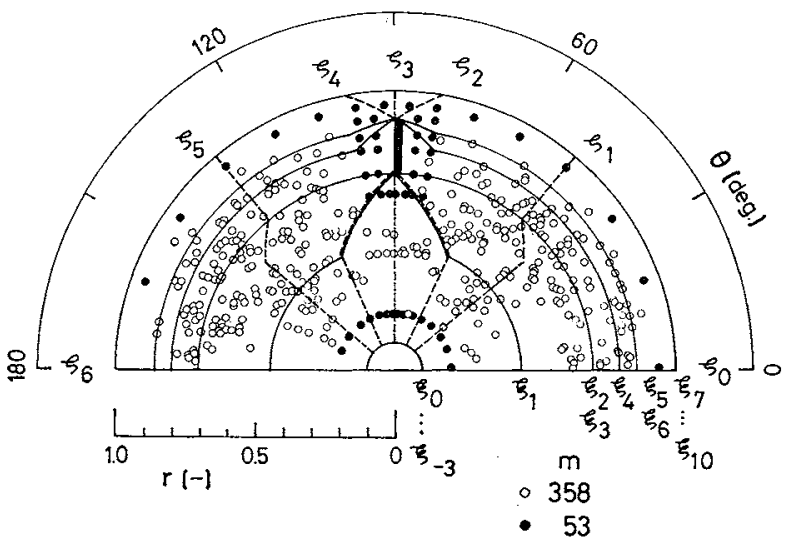

Fig. 7 Location of data points and grid used for correlation of experimental velocity distributions in anchor-agitated vessel

dinates of the positions of each small solid particle were interpolated with time by using a cubic polynomial spline.

$$
r=F_{r}(t), \theta=F_{\theta}(t), z=F_{z}(t)
$$

The velocity components can be obtained by differentiating the function of Eq. (37) with respect to time $t$ :

$$
V_{r}=F_{r}{ }^{\prime}(t), V_{\theta}=r \cdot F_{\theta}{ }^{\prime}(t), V_{z}=F_{z}{ }^{\prime}(t)
$$

where the functional forms of $F_{i}(t)$ and $F_{i}{ }^{\prime}(t)$ are given in the literature ${ }^{1,8)}$.

The parameter vector for all path lines of Eq. (37) are stored in a file in a computer with information on the region where each observed path line exists. Using this data file and Eqs. (37) and (38), the coordinates of all intersections of the path lines and a given plane, and the velocity components at the intersections are calculated and recorded in a file as the velocity data to be correlated. Since $V_{z}$ can be neglected as compared with $V_{r}$ and $V_{\theta}$ in an anchoragitated vessel, the data in several $(r, \theta)$ planes in 0.5 $<z<0.8$ are adopted as those in the same plane. The location of the data points (as indicated by 0 ) and a grid are shown in Fig. 7. It is very difficult to observe precisely a path line near solid boundaries by tracing a solid particle and this leads to a data shortage near the solid boundaries, as seen in the same figure. Some data from the numerical solution are added at these points as indicated by $(\bullet)$.

Figure 8 shows comparisons of experimental and correlation results. The tangential velocity distributions are drawn at $\theta=15^{\circ}, 45^{\circ}, 75^{\circ}, 105^{\circ}, 135^{\circ}$ and $165^{\circ}$, and the radial ones at $r=0.4,0.7$ and 0.9 , using the co-ordinate system fixed on the vessel wall. Excellent correlation results are obtained over the entire flow field.

\section{Conclusions}

A surface-fitting method with use of a cubic B- 


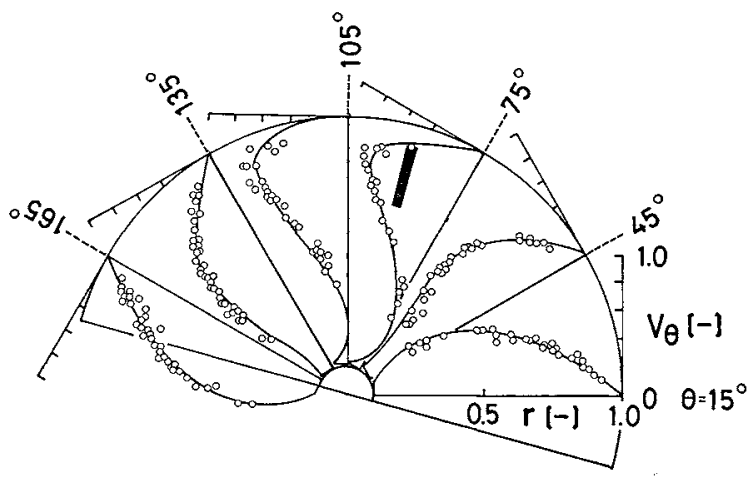

(a) tangential velocity distributions

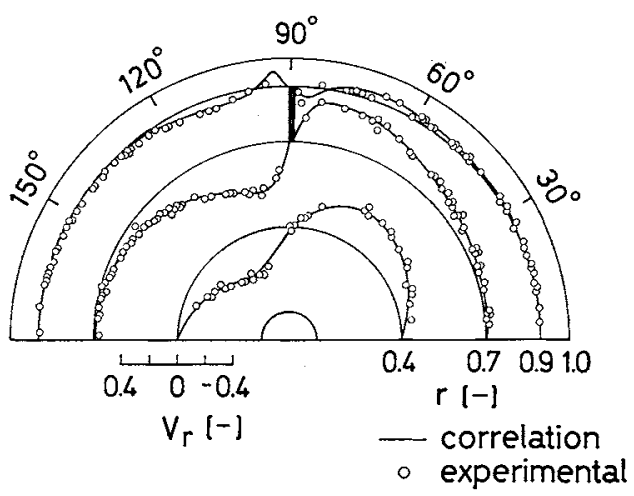

(b) radial velocity distributions

Fig. 8 Comparison of experimental and correlation results

spline function was applied to the correlation of the velocity distributions in an anchor-agitated vessel. The data for the path lines being available, this method has been proved to be of great use in the quantitative expression of the velocity throughout an agitated vessel.

Appendix The matrix $A$ and the column vector $d$ in Eq. (33)

The matrix $A$ takes the form of

$$
A=\left(\begin{array}{c}
A_{1,1} A_{1,2} A_{1,3} A_{1,4} \\
A_{2,1} A_{2,2} A_{2,3} A_{2,4} A_{2,5} \\
A_{3,1} A_{3,2} A_{3,3} A_{3,4} A_{3,5} A_{3,6} \\
A_{4,1} A_{4,2} A_{4,3} A_{4,4} A_{4,5} A_{4,6} A_{4,7} \\
A_{5,2} A_{5,3} A_{5,4} A_{5,5} A_{5,6} A_{5,7} A_{5,3} \\
\ldots \ldots \\
\ldots \ldots \\
A_{p+3, p} A_{p+3, p+1} A_{p+3, p+2} A_{p+3, p+3} A_{p+3, p+4} \\
A_{p+4, p+1} A_{p+4, p+2} A_{p+4, p+3} A_{p+4, p+4}
\end{array}\right)
$$

Here, each element $A_{j_{1}, j_{2}}$ is a rectangular matrix of the following form.

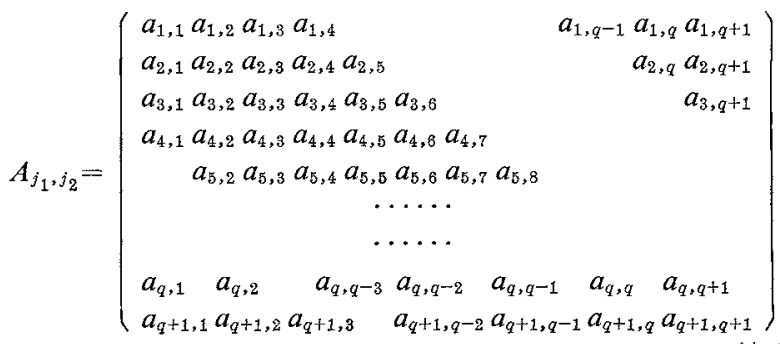

Each element $a_{k_{1}, k_{2}}$ in a matrix element $A_{j_{1}, j_{2}}$ is given as follows.

$$
a_{k_{1}, k_{2}}=\sum_{\left(r_{d}, \theta_{d}\right) \in\left\{\Omega_{\left(j_{1}, k_{1}\right)} \cap \Omega_{\left(j_{2}, k_{2}\right)}\right\}} M_{j_{1}}\left(r_{d}\right) N_{k_{1}}\left(\theta_{d}\right) M_{j_{2}}\left(r_{d}\right) N_{k_{2}}\left(\theta_{d}\right)
$$

In the equation, $\Omega_{(j, k)}$ consists of the regions $R_{f, g}$ where both of the B-splines, $M_{j}\left(r_{d}\right)$ and $N_{k}\left(\theta_{d}\right)$, are non-zero and is given as follows:

$$
\begin{gathered}
\Omega_{(j, k)}=\sum_{f=j \rightarrow 3}^{j} \sum_{g=k-3}^{k} R_{f, g} \\
(j=1,2, \cdots, p+1 \quad k=1,2, \cdots, q+1)
\end{gathered}
$$

From the periodic condition in $\theta$-direction (see Eqs. (17) and (18)), undefined regions, $R_{j,-2}, R_{j,-1}$ and $R_{j, 0}$, should be replaced by $R_{j, q-1}, R_{j, q}$ and $R_{j, q+1}$, respectively.

The column vector $\boldsymbol{d}$ is expressed as follows.

$$
\boldsymbol{d}=\left(d_{1,1}, d_{2,1}, \cdots, d_{j, k}, \cdots, d_{p+4, q+1}\right)^{T}
$$

where each element $d_{j, k}$ is given as follows.

$$
d_{j, k}=\sum_{\left(r_{d}, \theta_{d}\right) \in \Omega_{(j, k)}} M_{j}\left(r_{d}\right) N_{k}\left(\theta_{d}\right) V_{i, d}
$$

\section{Acknowledgement}

This work was supported by the Grant-in-Aid for Developmental Scientific Research (2) of the Ministry of Education, Science and Culture, Japan, Project No. 485232.

\section{Nomenclature}

$\begin{array}{ll}A & =\text { matrix defined by Eq. (A-1) } \\ A_{j_{1}, j_{2}} & =\text { element matrix of } A \text { matrix } \\ a_{k_{1}, k_{2}} & =\text { element of matrix } A_{j_{1}, j_{2}} \\ B_{n, j}(x) & =\text { B-spline of degree } n \\ c & =\text { column vector defined by Eq. (2) } \\ c_{j, k} & =\text { element of column vector } c \\ \boldsymbol{d} & =\text { column vector defined by Eq. (A-5) } \\ d_{j, k} & =\text { element of column vector } \boldsymbol{d} \text { defined by } \\ & \text { Eq. (A-6) }\end{array}$

$M_{j}(r), N_{k}(\theta)=$ normalized cubic B-spline defined by Eq. (5)

$m \quad=$ number of data points

$n \quad=$ degree of spline

$p, q=$ numbers of lines $\xi$ and $\zeta$ except additional lines

$Q \quad=$ sum of squares of residuals

$R_{j, k} \quad=$ region bounded by $\xi_{j-1}, \xi_{j}, \zeta_{k-1}$ and $\zeta_{k}$

$r \quad=$ dimensionless radial distance in polar co-ordinates (reduced by vessel radius)

$r_{b i}, r_{b o}=$ dimensionless radius of inner and outer tips of blade

$r_{d} \quad=$ dimensionless radial distance of datum point

$r_{s} \quad=$ dimensionless radius of shaft

$r_{w} \quad=$ dimensionless radius of vessel wall

$S_{i}(r, \theta, c)=$ approximating function

$t \quad=$ time

$V_{i} \quad=$ component of dimensionless velocity (reduced by maximum tangential velocity)

$V_{i, i}=$ component of dimensionless velocity data

$v_{i} \quad=$ velocity function

$\varepsilon_{\mathrm{av}}=$ average deviation of correlation values from true values

$=$ average error of data defined by Eq. (36)

$=$ data error

$=$ angle in polar co-ordinates 


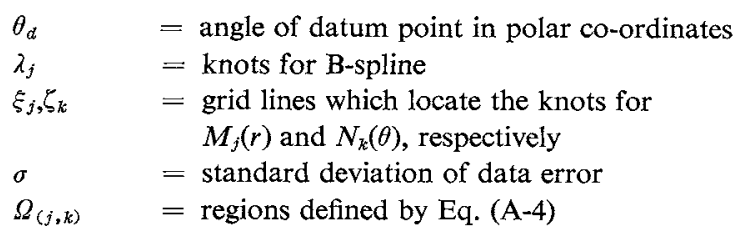

Literature Cited

1) Ahlberg, J. H., E. N. Nilson and J. L. Walsh: "The Theory of Splines and Their Applications", Academic Press, New York and London (1967).

2) de Boor, C.: J. Maths Phys., 41, 212 (1962).

3) Bourne, J. R. and H. Butler: Trans. Inst. Chem. Engrs., 47, T11 (1969).

4) Brebbia, C. A. and A. J. Ferrante: "Computational Methods for Solution of Engineering Problems", Crane, Russak \& Company, Inc., New York, U.S.A. (1978).

5) Carreau, P. J., I. Patterson and C. Y. Yap: Can. J. Chem. Eng., 54, 135 (1976).
6) Cox, M. G.: J. Inst. Maths. Applics, 10, 134 (1972).

7) Hayes, J. G. and J. Halliday: ibid., 14, 89 (1974).

8) Ichida, K. and F. Yoshimoto: "Spline Functions and Applications", Kyoiku Shuppan Co. Ltd., Tokyo (1979).

9) Kamiwano, M., T. Motoyoshi, F. Shirasaka and T. Wakabayashi: Kagaku Kogaku Ronbunshu, 6, 105 (1980).

10) Kuriyama, M., H. Inomata, K. Arai and S. Saito: AIChE J., 28, 385 (1982).

11) Murakami, Y., K. Fujimoto, T. Shimada, A. Yamada and K. Asano: J. Chem. Eng. Japan, 5, 297 (1972).

12) Peters, D. C. and J. M. Smith: Trans. Inst. Chem. Engrs., 45, T360 (1967).

13) idem: Can. J. Chem. Eng., 47, 268 (1969).

14) Rice, J. R.: "The Approximation of Functions", AddisonWesley, Reading, Massachusetts, (1969).

15) Schoenberg, I. J. and A. Whitney: Trans. Amer. Math. Soc., 74, 246 (1953).

16) Yoshimoto, F., K. Ichida and T. Kiyono: Jyoho Shori, 18, 128 (1977).

\title{
Short Communications
}

\section{AN OPTICAL PROBE TO DETECT BOTH BUBBLES AND SUSPENDED PARTICLES IN A THREE-PHASE FLUIDIZED BED}

\author{
MASARU ISHIDA AND HITOSHI TANAKA \\ Research Laboratory of Resources Utilization, Tokyo Institute of Technology, Yokohama 227
}

Optical fiber probes have widely been applied to measure the size and rising velocity of bubbles as well as the direction and velocity of movement of particles in a gas-solid fluidized bed ${ }^{2,3,5)}$. In these cases, the probe was made of multiple fibers; one is used as light projector and the others as receivers of the light penetrating through the bed $^{2}$ or reflected on the particles around the tip of the probe ${ }^{3,5)}$.

For gas-liquid systems, Abuaf et al. ${ }^{11}$ proposed a total-reflection probe to detect gas bubbles. They set two optical fibers together, fused their ends, and made an arrow-type head to obtain total reflection when the probe was immersed in the bubble phase. Recently, the authors ${ }^{4}$ ) have shown that a single-fiber probe of quite simple shape, whose tip was sharpened like that of Abuaf et al., worked satisfactorily. They used it to observe the behavior of bubbles in a threephase fluidized bed. However, these probes were unable to detect the movement of particles in the bed.

\footnotetext{
Received January 9, 1982. Correspondence concerning this article should be addressed to $\mathbf{M}$. Ishida.
}

The purpose of this paper is to propose a new optical probe which can give characteristic signals for both bubbles and particles in a three-phase fluidized bed.

\section{Structure of Optical Probes}

The typical reflective probe used in a fluidized bed (probe A) and the total-reflection probe (probe B) of Abuaf et al. are shown schematically in Figs. 1(a) and (b). Both probes consist of two fibers, one of which is a light projector. For probe $\mathrm{A}$, the other fiber receives the light reflected on the surface of the particles around the tip of the probe, whereas for probe $B$ it receives the light reflected twice on the conical interface between the quartz glass and the fluid phase.

Figures 2 (a) and (b) show new probes. They are made of a single optical fiber with two others connected. The single fiber has the dual functions of projecting light and receiving its reflection. Be- 